\title{
Produção do inhame em solo adubado com fontes e doses de matéria orgânica
}

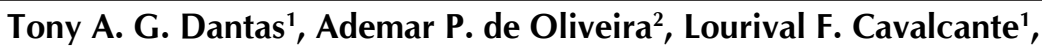 \\ Damiana F. da S. Dantas', Natália V. da S. Bandeira ${ }^{1}$ \& Stênio A. G. Dantas ${ }^{1}$
}

\begin{abstract}
RESUMO
Objetivou-se neste trabalho avaliar o comportamento do inhame, cultivar Da Costa, adubado com fontes e doses de matéria orgânica. O delineamento experimental foi em blocos casualizados distribuídos em esquema fatorial $6 \times 2$, correspondendo a seis doses $\left(0,6,12,18,24\right.$ e $\left.30 \mathrm{tha}^{-1}\right)$ e duas fontes de matéria orgânica (esterco bovino e caprino), em três repetições. O comprimento de túberas aumentou de forma linear com valor máximo de $27,5 \mathrm{~cm}$ na dose de $30 \mathrm{t} \mathrm{ha}^{-1}$ de esterco caprino e o esterco bovino proporcionou túberas com comprimento médio de 23,64 cm. O diâmetro foi de 9,1 e 9,3 cm nas doses de 20 e 21,6 tha-1 de esterco caprino e bovino, respectivamente. A máxima massa média de túberas de 1,4 kg foi obtida com a dose 17,4 tha-1 de esterco caprino e, em função do fornecimento das doses de esterco bovino obteve-se o valor médio de $1,11 \mathrm{~kg}$. A dose de 20,9 tha-1 de esterco caprino proporcionou a máxima produtividade comercial de túberas de $14 \mathrm{t} \mathrm{ha}^{-1} \mathrm{e}$ produtividade média de $10,1 \mathrm{t} \mathrm{ha}^{-1}$ com o uso de esterco bovino. $\mathrm{O}$ esterco caprino foi superior ao bovino e a dose em torno de $20 \mathrm{t} \mathrm{ha}^{-1}$ de ambos os estercos promove os maiores valores nas características avaliadas.
\end{abstract}

Palavras-chave: Dioscorea cayennensis, adubação orgânica, produtividade de túberas

\section{Production of yam in a soil fertilized with different sources and rates of organic matter}

\begin{abstract}
The objective of this study was to evaluate the behavior of yam, variety Da Costa, fertilized with different sources and rates of organic matter. The experimental design was a randomized complete blocks distributed in a factorial $2 \times 6$, corresponding to six rates $\left(0,6,12,18,24\right.$ and $\left.30 \mathrm{t} \mathrm{ha}^{-1}\right)$ and two sources of organic matter (cattle and goat manure), with three replications. The length of tubers increased linearly with maximum value of $27.5 \mathrm{~cm}$ at a rate of $30 \mathrm{t} \mathrm{ha}^{-1}$ of goat manure and the bovine manure provided tubers with mean length of $23.64 \mathrm{~cm}$. The diameter was of 9.1 and $9.3 \mathrm{~cm}$ in rates of 20 and $21.6 \mathrm{tha}^{-1}$ of goat and bovine manure, respectively. The maximum mean mass of $1.4 \mathrm{~kg}$ was obtained with the rate $17.4 \mathrm{t}$ $\mathrm{ha}^{-1}$ of goat manure and in function of rates of bovine manure the mean value obtained was $1.11 \mathrm{~kg}$. A rate of $20.9 \mathrm{t} \mathrm{ha}^{-1}$ of goat manure provided the maximum commercial productivity of tubers of $14 \mathrm{t} \mathrm{ha}^{-1}$, while mean productivity of $10.1 \mathrm{t} \mathrm{ha}^{-1}$ was obtaines with the use of bovine manure. The goat manure is better than bovine manure and the rate around of $20 \mathrm{tha}^{-1}$ of both the manures promoted the highest values of the evaluated characteristics.
\end{abstract}

Key words: Dioscorea cayennensis, organic fertilization, productivity of tubers

${ }^{1}$ Departamento de Fitotecnia e Ciências Ambientais/UFPB. CEP 58397-000, Areia, PB. Fone: (83) 3362-2300. E-mail: tagdantas@yahoo.com.br; lofeca@cca.ufpb.br; damyagro@hotmail.com; natalia.vitaldasilva@yahoo.com.br; stenioandrey@bol.com.br

${ }^{2}$ Departamento de Fitotecnia e Ciências Ambientais/UFPB. Bolsista de produtividade em pesquisa CNPq. E-mail: ademar@cca.ufpb.br 


\section{INTRODUÇÃO}

O inhame (Dioscorea cayennensis), pertence ao gênero Dioscorea e à família das Dioscoreaceas, que possui mais de 600 espécies dentre as quais 60 são cultivadas para fins farmacêuticos e/ou alimentícios. É uma planta herbácea com hábito de crescimento determinante, anual ou perene e propagada vegetativamente por meio da fragmentação de tubérculos. No Brasil se plantam inhames das espécies Dioscorea cayennensis Lam. (Inhame da costa) e Dioscorea alata L. (São Tomé ou cará) cultivados principalmente na região Nordeste, nos estados de Pernambuco e Paraíba, onde encontram condições edafoclimáticas favoráveis ao seu cultivo (Siqueira, 2011; Silva et al., 2012).

O cultivo do inhame é de grande importância socioeconômica para a região Nordeste por ser frequente na dieta regional. Destaca-se pelo valor agregado de suas túberas em relação a outras fontes de carboidrato, como batata doce e mandioca, e pela alta necessidade de mão-de-obra durante seu ciclo, principalmente na colheita, gerando emprego e renda. Do ponto de vista nutricional sua túbera é útil para a saúde humana devido aos teores de minerais $(\mathrm{Ca}, \mathrm{P} \mathrm{e} \mathrm{Fe})$, carboidratos, aminoácidos essenciais, provitamina $\mathrm{A}$, provitamina $\mathrm{D}$, vitaminas $\mathrm{C}$ e do complexo B, além de suas propriedades medicinais que garantem o uso na farmacologia com destaque na síntese de cortisona e hormônios esteroides (Mesquita, 2002; Oliveira et al., 2011).

Segundo Santos et al. (2009) apesar da importância socioeconômica que o inhame representa para a região Nordeste, sobretudo para o estado da Paraíba, sua produtividade é ainda considerada baixa, de $10.500 \mathrm{~kg} \mathrm{ha}^{-1}$, em função de vários fatores, como: baixa fertilidade dos solos das zonas produtoras, baixo nível técnico dos produtores rurais, problemas fitossanitários e indisponibilidade de túberas semente de boa qualidade. Diby et al. (2011) destacam a fertilidade do solo como a principal limitação para o aumento da produtividade do inhame e, de acordo com O'Sullivan \& Ernest (2007), as túberas de inhame têm a capacidade de extrair grandes quantidades de nutrientes do solo ocasionando redução dos seus teores. Contudo, a adubação orgânica adequada é essencial para elevar seu potencial produtivo, melhorar a disponibilidade de nutrientes e aumentar a produtividade da cultura.

Dentre os adubos orgânicos disponíveis para fertilização do solo e para a cultura do inhame, se destacam o esterco bovino e o caprino, que promovem melhorias na fertilidade do solo pelo aumento da capacidade de troca catiônica e liberação de nutrientes, além de proporcionar melhores condições físicas ao solo, como agregação das partículas, estruturação e estabilidade do solo, melhora a capacidade de retenção de água e favorece a fauna microbiológica; esses adubos também proporcionam acúmulo de nitrogênio orgânico ao solo (Tejada et al., 2008; Borchartt et al., 2011).

O uso da matéria orgânica é uma alternativa amplamente adotada para o suprimento de nutrientes em áreas de agricultura familiar na região semiárida e no agreste do Nordeste do Brasil (Menezes \& Salcedo, 2007). No inhame, o emprego de matéria orgânica tem sido prática constante pelos produtores. Segundo Silva et al. (2012), apenas a adubação orgânica atende às exigências nutricionais do inhame; entretanto, informações sobre fontes e quantidades de matéria orgânica a serem aplicadas e seus efeitos no rendimento e na produtividade de túberas, ainda são limitadas e conflitantes. Portanto, objetivouse avaliar o desenvolvimento de túberas e a produtividade do inhame adubado com diferentes doses e fontes de matéria orgânica.

\section{Material e Métodos}

O experimento foi conduzido de fevereiro a setembro de 2009, em condições de campo, no Setor de Olericultura do Departamento de Fitotecnia do Centro de Ciências Agrárias (CCA) da Universidade Federal da Paraíba (UFPB), no município de Areia, PB, na Microrregião do Brejo Paraibano, com altitude de $574 \mathrm{~m}$. Pela classificação bioclimática de Koppen, o clima é do tipo As' (quente e úmido) com chuva durante o período outono-inverno (Brasil, 1972). Durante o período foram coletados dados de precipitação e temperatura, na estação meteorológica do Instituto Nacional de Meteorologia (INMET) no CCA/UFPB (Figura 1), localizada a aproximadamente $3 \mathrm{~km}$ do local do experimento.

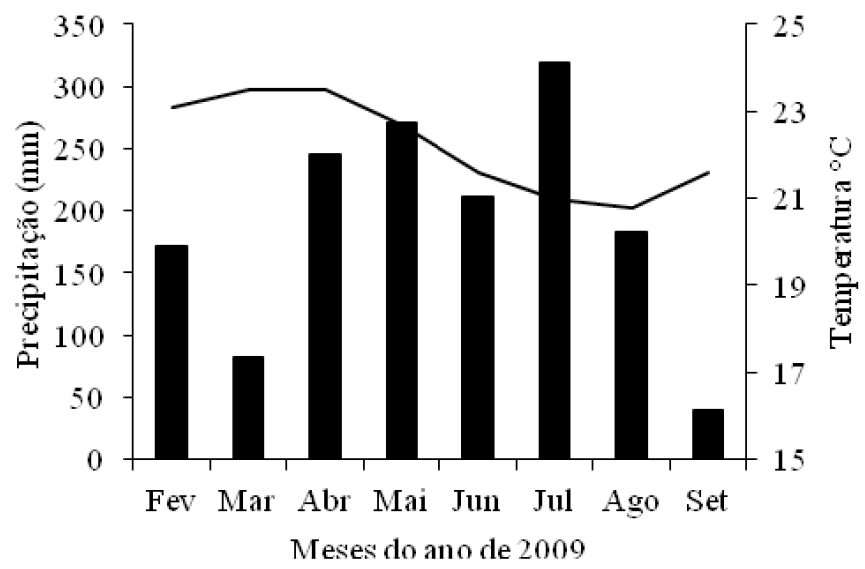

Figura 1. Dados de precipitação ( ) e temperatura (-) no período de fevereiro a setembro de 2009, obtidos na estação meteorológica do INMET/CCA/UFPB

O solo da área experimental foi classificado como Neossolo Regolítico Psamítico típico, textura franco-arenosa (EMBRAPA, 2006). As análises químicas e físicas do solo na camada de 0-20 $\mathrm{cm}$ foram realizadas de acordo com metodologia da EMBRAPA (1997) pelo Laboratório de Química e Fertilidade do Solo do Departamento de Solos e Engenharia Rural (DSER) da UFPB. Os resultados das análises químicas foram: $\mathrm{pH}$ em água $(1: 2,5)=$ 6,$48 ; \mathrm{P}\left(\mathrm{mg} \mathrm{dm}^{-3}\right)=85,41 ; \mathrm{K}^{+}\left(\mathrm{mg} \mathrm{dm}^{-3}\right)=95,05 ; \mathrm{Na}^{+}\left(\mathrm{cmol} \mathrm{dm}^{-}\right.$ $\left.{ }^{3}\right)=0,22 ; \mathrm{H}^{+}+\mathrm{Al}^{+3}\left(\mathrm{cmol}_{\mathrm{c}} \mathrm{dm}^{-3}\right)=2,39 ; \mathrm{Al}^{+3}\left(\mathrm{cmol}_{\mathrm{c}} \mathrm{dm}^{-3}\right)=0,00$; $\mathrm{Ca}^{+2}\left(\mathrm{cmol}_{\mathrm{c}} \mathrm{dm}^{-3}\right)=2,35 ; \mathrm{Mg}^{+2}\left(\mathrm{cmol}_{\mathrm{c}} \mathrm{dm}^{-3}\right)=1,15 ; \mathrm{SB}\left(\mathrm{cmol}_{\mathrm{c}}\right.$ $\left.\mathrm{dm}^{-3}\right)=6,15$; CTC $\left(\mathrm{cmol}_{\mathrm{c}} \mathrm{dm}^{-3}\right)=8,54$ e Matéria orgânica $(\mathrm{g}$ $\left.\mathrm{dm}^{-3}\right)=8,79$. Para a análise física foi o seguinte: areia grossa $(\mathrm{g}$ $\left.\mathrm{kg}^{-1}\right)=672$; areia fina $\left(\mathrm{g} \mathrm{kg}^{-1}\right)=125$; silte $\left(\mathrm{g} \mathrm{kg}^{-1}\right)=126$; argila $\left(\mathrm{g} \mathrm{kg}^{-1}\right)=77$; densidade do solo $\left(\mathrm{kg} \mathrm{dm}^{-3}\right)=1,22$; densidade de partículas $\left(\mathrm{kg} \mathrm{dm}^{-3}\right)=2,66$; porosidade total $\left(\mathrm{m}^{3} \mathrm{~m}^{-3}\right)=0,54 \mathrm{e}$ classe textural $=$ areia franca.

$\mathrm{O}$ delineamento experimental utilizado foi de blocos casualizados, com três repetições, em esquema fatorial $6 \mathrm{x}$ 
2 , correspondendo a seis doses $\left(0,6,12,18,24\right.$ e $\left.30 \mathrm{t} \mathrm{ha}^{-1}\right) \mathrm{e}$ a duas fontes de matéria orgânica (esterco bovino e caprino). As parcelas foram constituídas de 20 plantas distribuídas no espaçamento de 1,2 x 0,6 m. Antes da instalação dos tratamentos foram coletadas amostras das duas fontes de matéria orgânica e levadas ao Laboratório de Química e Fertilidade do Solo do DSER/CCA/UFPB, para análise e determinação do teor de umidade das fontes de matéria orgânica, para serem consideradas e corrigidas nos cálculos de adubação (Tabela 1), segundo metodologia da EMBRAPA (1997).

Tabela 1. Características químicas dos estercos utilizados no experimento

\begin{tabular}{lcr}
\hline Atributos químicos & \multicolumn{3}{c}{ Esterco } \\
\cline { 2 - 3 } $\mathrm{pH}$ em água $(1: 2,5)$ & Bovino & Caprino \\
$\mathrm{C}\left(\mathrm{g} \mathrm{kg}^{-1}\right)$ & 8,74 & 8,93 \\
$\mathrm{M} .0\left(\mathrm{~g} \mathrm{dm}^{-3}\right)$ & 200,00 & 175,40 \\
$\mathrm{P}\left(\mathrm{mg} \mathrm{dm}^{-3}\right)$ & 344,80 & 302,40 \\
$\mathrm{~K}^{+}\left(\mathrm{mg} \mathrm{dm}^{-3}\right)$ & 690,42 & 499,28 \\
$\mathrm{Ca}^{2+}\left(\mathrm{cmol}_{\mathrm{c}} \mathrm{dm}^{-3}\right)$ & 2826,00 & 3624,00 \\
$\mathrm{Mg}^{2+}\left(\mathrm{cmol}_{\mathrm{c}} \mathrm{dm}^{-3}\right)$ & 9,20 & 12,70 \\
$\mathrm{Na}^{+}\left(\mathrm{cmol}_{\mathrm{c}} \mathrm{dm}^{-3}\right)$ & 2,80 & 8,00 \\
Relação C:N $_{\text {Umidade (\%) }}$ & 6,61 & 8,31 \\
& $15: 1$ & $14: 1$ \\
& 5,00 & 5,00 \\
\hline
\end{tabular}

O preparo do solo constou de aração e gradagem para propiciar condições favoráveis ao plantio e desenvolvimento das túberas, em seguida e de forma manual foram confeccionados leirões com $0,5 \mathrm{~m}$ de altura. A adubação constou apenas do fornecimento das fontes e doses de matéria orgânica, descritas anteriormente, as quais foram distribuídas por cova, sete dias antes do plantio.

O plantio foi realizado em fevereiro de 2009 empregandose porções de túberas semente da cultivar de inhame da Costa com peso médio de aproximadamente $250 \mathrm{~g}$ enterradas a 10 $\mathrm{cm}$ de profundidade do topo do leirão. Durante a condução do experimento foram executadas capinas manuais com o auxílio de enxadas, para manter a área livre de plantas espontâneas. Por ocasião das capinas foram realizadas amontoas, com o objetivo de manter os leirões bem formados e proteger as túberas contra o efeito dos raios solares. Para orientação do crescimento da planta foi adotado o sistema de tutoramento tradicional (vara simples de bambu), medindo aproximadamente 1,80 $\mathrm{m}$ de altura. Não houve incidência de pragas e doenças que comprometesse a produção da cultura.

A colheita foi realizada aos sete meses após o plantio (setembro/2009), considerada colheita precoce ou capação, caracterizada pelo término da floração com secamento das flores. Foram avaliados o comprimento e o diâmetro das túberas comerciais com o auxílio de régua e paquímetro digital, respectivamente e consideradas túberas comerciais aquelas com massa entre 1,5 e 3,0 kg. A massa média foi determinada pela relação estabelecida entre a produção de túberas da parcela e o número de túberas comerciais, expresso em $\mathrm{kg}$. A produtividade comercial correspondeu à massa de todas as túberas comerciais colhidas na parcela transformando os resultados para $t \mathrm{ha}^{-1}$.

Os resultados foram submetidos à análise de variância e as médias comparadas pelo teste Tukey, a 0,05 de probabilidade. Foram realizadas, também, análises de regressão polinomial para comparar os efeitos das doses de estercos aplicadas selecionando-se para expressar os resultados, o modelo significativo de maior ordem e com maior coeficiente de determinação. Nas análises estatísticas foi empregado o programa software $\mathrm{SAEG}^{\circledR}$ (SAEG, 2000).

\section{Resultados e Discussão}

Houve efeitos significativos $(p \leq 0,05)$ das doses de esterco bovino e caprino para o comprimento, diâmetro, massa média e produtividade de túberas comerciais.

O comprimento das túberas comerciais de inhame aumentou de forma linear com o aumento das doses de esterco caprino atingindo valor máximo de $27,5 \mathrm{~cm}$ na dose de $30 \mathrm{tha}^{-1}$. Quando adubado com esterco bovino não se observou efeito de doses sendo o comprimento médio de túberas de 23,64 $\mathrm{cm}$ (Figura 2). Esses valores são semelhantes aos obtidos por Silva (2010), que trabalhando com esterco bovino verificou comprimento máximo de túberas de $26 \mathrm{~cm}$, na dose estimada de $29 \mathrm{t} \mathrm{ha}^{-1}$. Oliveira et al. (2007) verificaram comprimento de túberas de $32 \mathrm{~cm}$ com $70 \mathrm{~kg}$ de $\mathrm{N} \mathrm{ha}^{-1} \mathrm{e} 12 \mathrm{t} \mathrm{ha}^{-1}$ de esterco bovino fornecido no plantio. Santos et al. (2009), não constataram diferenças significativas entre os tratamentos estudando fertilização do solo com macro e micronutrientes, mas obtiveram valor médio de comprimento de túberas de $25,63 \mathrm{~cm}$, portanto, o esterco bovino e caprino proporciona aumento no comprimento do inhame provavelmente por estimular o desenvolvimento das túberas.

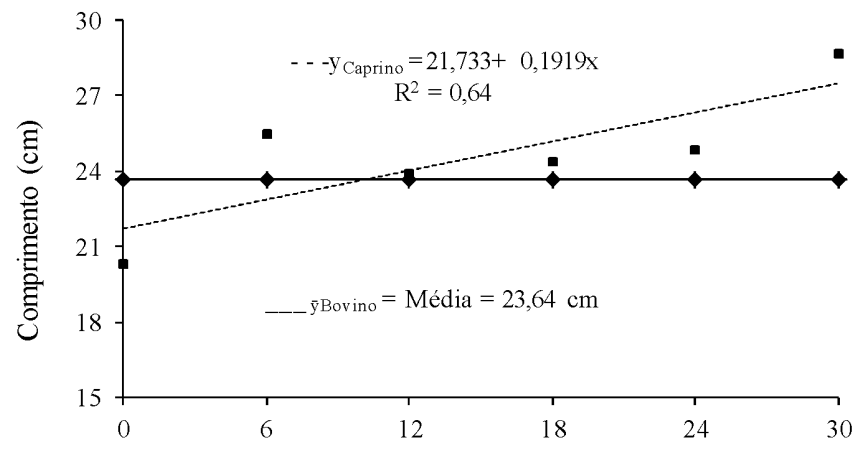

Doses de estercos $\left(\mathrm{t} \mathrm{ha}^{-1}\right)$

Figura 2. Comprimento de túberas comerciais de inhame adubado com estercos bovino (-) e caprino $(--)$

Os diâmetros máximos de túberas comerciais foram 9,3 e $9,1 \mathrm{~cm}$ estimados com as doses de 20 e $21,6 \mathrm{t} \mathrm{ha}^{-1}$ de esterco caprino e bovino, respectivamente (Figura 3). De acordo com Oliveira et al. (2007), esses diâmetros são considerados adequados para o comércio interno e externo, o que pode indicar que o fornecimento de esterco bovino e caprino atende às exigências nutricionais do inhame melhorando a qualidade comercial das túberas. Esses valores são superiores aos encontrados por Silva (2010), que obteve túberas com diâmetro médio de $7,9 \mathrm{~cm}$ de diâmetro em função das doses de esterco bovino. Os valores obtidos para o diâmetro de túberas refletem a importância do uso da matéria orgânica para melhoria das condições físicas do solo proporcionando melhor 


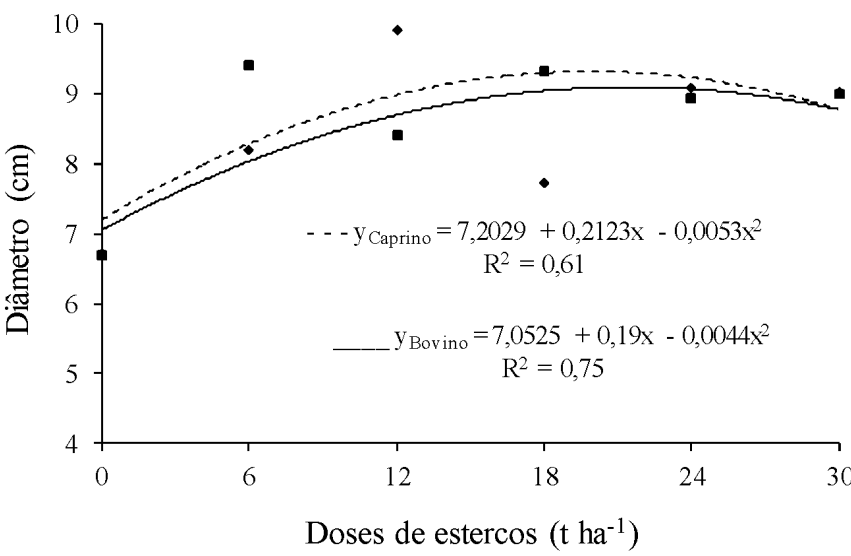

Figura 3. Diâmetro de túberas comerciais de inhame adubado com esterco bovino (-) e caprino (- - -)

ambiente para o desenvolvimento das túberas. Borchartt et al. (2011) constataram, trabalhando com batata, que o aumento dos teores de matéria orgânica do solo aumenta a liberação de nutrientes, principalmente nitrogênio, que vai interferir diretamente no aumento dos tubérculos.

O valor máximo de massa média de túberas comerciais foi $1,4 \mathrm{~kg}$ obtido na dose $17,4 \mathrm{tha}^{-1}$ de esterco caprino, porém com esterco bovino não houve diferenças entre doses, sendo a massa média de $1,11 \mathrm{~kg}$ (Figura 4). De acordo com Oliveira et al. (2007), esses valores se encontram abaixo daqueles recomendados para túberas comerciais de inhame que se situam entre 1,5 a $2,0 \mathrm{~kg}$. Os valores obtidos são inferiores aos encontrados por Silva et al. (2012) que obtiveram túberas de inhame com massa superior a 2,0 kg na dose de $30 \mathrm{t} \mathrm{ha}^{-1} \mathrm{de}$ esterco bovino e Oliveira et al. (2011) trabalhando com doses de fósforo, obtiveram túberas com 1,94 $\mathrm{kg}$ na dose de $245 \mathrm{~kg}$ ha ${ }^{-1}$ de $\mathrm{P}_{2} \mathrm{O}_{5}$ e com $15 \mathrm{t} \mathrm{ha}^{-1}$ de esterco bovino fornecido em cobertura, porém esses valores estão próximos aos de Oliveira et al. (2007), trabalhando com adubação nitrogenada e com o fornecimento de $12 \mathrm{t} \mathrm{ha}^{-1}$ de esterco bovino no plantio, obtiveram valor máximo estimado de $1,5 \mathrm{~kg}$ na dose de 120 $\mathrm{kg} \mathrm{ha}^{-1}$ de N.

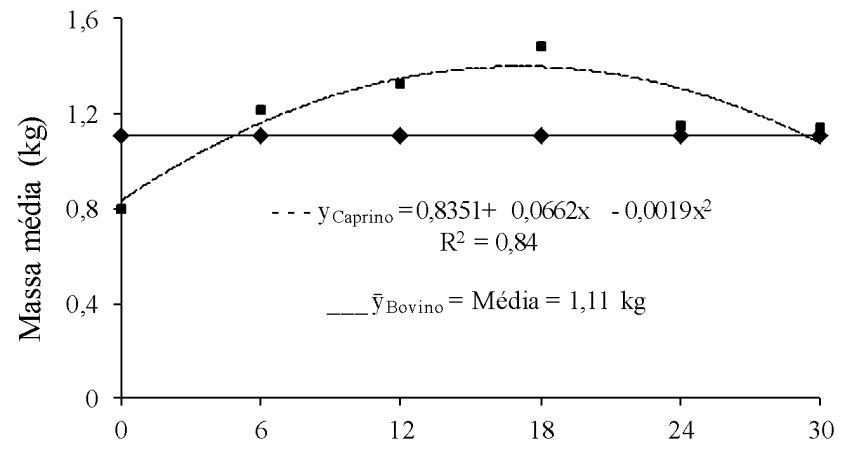

Doses de estercos $\left(\mathrm{t} \mathrm{ha}^{-1}\right)$

Figura 4. Massa média de túberas comerciais de inhame adubado com esterco bovino (-) e caprino $(--)$

É provável que os valores de massa média de túberas sejam inferiores aos obtidos por outros autores devido às condições climáticas em que o trabalho foi realizado, pois a fase de formação das túberas se situa após os 140 dias a partir do plantio e no ano de 2009 registrou-se uma precipitação acumulada de mais de $1500 \mathrm{~mm}$ durante o período do experimento (Figura 1), portanto, houve diminuição da temperatura o que, possivelmente, reduziu a fotossíntese e o acúmulo de assimilados atrasando a formação das túberas (Diby et al., 2011).

A produtividade máxima de túberas comerciais foi de $14 \mathrm{t} \mathrm{ha}^{-1}$ estimada na dose de 20,9 $\mathrm{t} \mathrm{ha}^{-1}$ de esterco caprino, porém o esterco bovino não se ajustou ao modelo de regressão apresentando valor médio de 10,1 t ha-1 de túberas (Figura 5). As produtividades obtidas foram consideradas baixas quando comparadas a de 20,3 tha-1 obtida na dose de 19,2 $\mathrm{t} \mathrm{ha}^{-1} \mathrm{de}$ esterco bovino (Silva et al., 2012) e a de $18 \mathrm{t} \mathrm{ha}^{-1}$ de túberas na dose de 13,3 tha ${ }^{-1}$ de esterco bovino associada a 100-120-60 $\mathrm{kg} \mathrm{ha}^{-1}$ de N- $\mathrm{P}_{2} \mathrm{O}_{5}-\mathrm{K}_{2} \mathrm{O}$ (Oliveira et al., 2001); entretanto, foram superiores à produtividade máxima de $11,8 \mathrm{t} \mathrm{ha}^{-1}$ obtida por Oliveira et al. (2011), na dose de $266 \mathrm{~kg} \mathrm{ha}^{-1} \mathrm{de}_{2} \mathrm{O}_{5}$, aplicado com 15 tha $^{-1}$ de esterco bovino e $70 \mathrm{~kg} \mathrm{ha}^{-1} \mathrm{de} \mathrm{K}_{2} \mathrm{O}$ e de $80 \mathrm{~kg} \mathrm{ha}^{-1} \mathrm{de}$ $\mathrm{N}$ em adubação de cobertura e além do fornecimento parcelados em partes iguais aos 60 e 90 dias após o plantio.

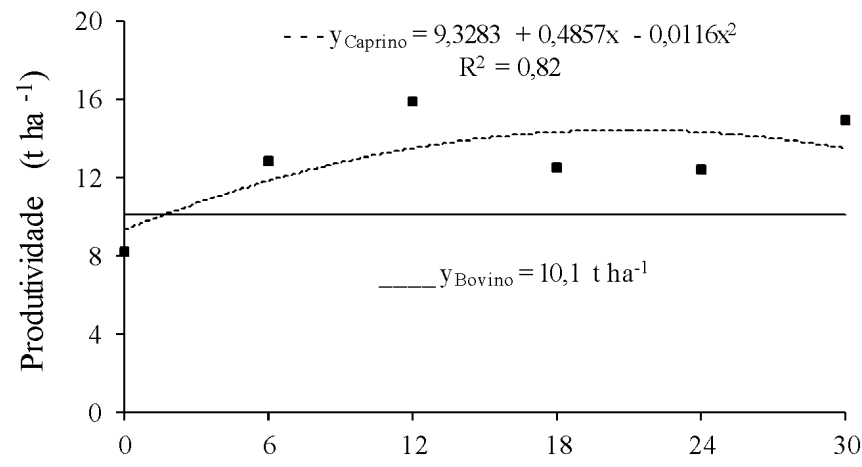

Doses de estercos $\left(\mathrm{t} \mathrm{ha}^{-1}\right)$

Figura 5. Produtividade comercial de túberas de inhame adubado com esterco bovino (-) e caprino $(--)$

Os efeitos positivos da adição de matéria orgânica sobre a produtividade de inhame se devem, além do fornecimento de nutrientes, à sua ação na melhoria da capacidade de troca de bases, promovendo maior disponibilidade de nutrientes para a planta, durante um longo período. Esses efeitos são mais acentuados em solos de baixo teor de matéria orgânica, como ocorre no presente trabalho $\left(8,79 \mathrm{~g} \mathrm{dm}^{-3}\right)$, além de melhorar as características físicas do solo proporcionando um ambiente mais propício ao desenvolvimento das túberas (Oliveira et al., 2001). De acordo com Diby et al. (2011), o potássio é o segundo elemento mineral mais absorvido pelas Dioscorea sp., o teor de potássio encontrado no esterco caprino é $28 \%$ superior ao teor encontrado na composição do esterco bovino (Tabela 2), o que também pode ter influenciado na maior resposta da produtividade a essa fonte de matéria orgânica.

\section{ConclusõES}

1. A adubação orgânica com esterco caprino foi superior à do esterco bovino nas características avaliadas no inhame (Dioscorea cayennensis). 
2. A dose em torno de $20 \mathrm{t} \mathrm{ha}^{-1}$ de ambos os estercos promove os maiores valores de diâmetro, massa média e produtividade de túberas comerciais de inhame.

\section{Literatura Citada}

Borchartt, L.; Silva, I. F.; Santana, E, O.; Sousa, C.; Ferreira, L. E. Adubação orgânica da batata com esterco bovino no município de Esperança, PB. Revista Ciência Agronômica, v.42, p.482-487, 2011.

Diby, L. N.; Hgaza, V. K.; Tié, T. B.; Carsky, R.; Girardin, O.; Assa, A. Mineral nutrients uptake and partitioning in Dioscorea alata and Dioscora rotundata. Journal of Applied Biosciences, v.38, p.2531-2539, 2011.

Brasil. Ministério da Agricultura. Levantamento exploratório - Reconhecimentos de solos do estado da Paraíba. Rio de Janeiro: MA/SUDENE, v.15, 1972. 670p. Boletim Técnico

EMBRAPA - Empresa Brasileira de Pesquisa Agropecuária. Centro Nacional de Pesquisa de solos. Manual de métodos de análise de solo. 2.ed. Rio de Janeiro: EMBRAPA, 1997. 212p.

EMBRAPA - Empresa Brasileira de Pesquisa Agropecuária. Centro Nacional de Pesquisa de Solos. Sistema brasileiro de classificação de solos. Brasília: Embrapa Solos, 2006. 412p.

Menezes, R. S. C.; Salcedo. I. H. Mineralização de N após incorporação de adubos orgânicos em um Neossolo Regolítico cultivado com milho. Revista Brasileira de Engenharia Agrícola e Ambiental, v.11, p.361-367, 2007.

Mesquita, A. S. Inhame e taro: Cenários dos mercados internacional, brasileiro e baiano. Bahia Agrícola, v.5, p.54-64, 2002.

Oliveira, A. N. P; Oliveira, F. A.; Sousa, L. C.; Oliveira, A. P.; Silva, J. A.; Silva, D. F.; Silva, N. V.; Santos, R. R. Adubação fosfatada em inhame em duas épocas de colheita. Horticultura Brasileira v. 9, p.456-460, 2011.
Oliveira, A. P.; Barbosa, L. J. N.; Pereira, W. E.; Silva, J. E. L.; Oliveira, A. N. P. Produção de rizóforos comerciais de inhame em função de doses de nitrogênio. Horticultura Brasileira, v.25, p.73-76, 2007.

Oliveira, A. P.; Freitas Neto, P. A.; Santos E. S. Produtividade do inhame, em função de fertilização orgânica e mineral e de épocas de colheita. Horticultura Brasileira, v.19, p.144147, 2001.

O'Sullivan, J. N.; Ernest, J. Nutrient deficiencies in lesser yam (Dioscorea esculenta) characterized using constant-water table sand culture. Journal of Plant Nutrition and Soil Science, v.170, p.273-282. 2007.

SAEG - Sistema para análise estatística, versão 8.0. Viçosa: Fundação Artur Bernardes, 2000.

Santos, E. S.; Macêdo, L. S.; Matias, E. C.; Barbosa, M. M. Resposta da cultura do inhame à fertilização com macro e micronutrientes em um Argissolo Vermelho-Amarelo Distrófico arênico, Tecnologia \& Ciência Agropecuária, v.3, p.39-46, 2009.

Silva, J. A. Rendimento e qualidade do inhame adubado com esterco bovino e biofertilizante. Areia: UFPB, 2010. 75p. Tese Doutorado

Silva, J. A.; Oliveira, A. P.; Alves, G. S.; Cavalcante, L. F.; Oliveira, A. N. P.; Araújo, M. A. M. Rendimento do inhame adubado com esterco bovino e biofertilizante no solo e na folha. Revista Brasileira de Engenharia Agrícola e Ambiental, v.16, p.253-257, 2012.

Siqueira, M. V. B. M. Yam: A neglected and underutilized crop in Brazil. Horticultura Brasileira, v.29, p.16-20, 2011.

Tejada, M.; Gonzalez, J. L.; García-Martínez, A. M.; Parrado, J. Effects of different green manures on soil biological properties and maize yield. Bioresource Technology, v.99, p.1758-1767, 2008. 